\title{
A CORPORATE STRATEGY FOR IMPLEMENTING PROCESS SIMULATION
}

\author{
Douglas J. Payne \\ Nandu N. Thondavadi \\ Phil Francis \\ Corporate Technology Center \\ Square D Company \\ 1415 S. Roselle Road \\ Palatine, Illinois 60067
}

\begin{abstract}
Simulation modeling of complex manufacturing systems has been recognized as a critical link to a successful CIM strategy. Square D Company has embarked on an ambitious program of institutionalizing modern methods of manufacturing practices in its plants worldwide by forming the Corporate Technology Center (CTC), a center of excellence with the charter to provide product and process related advanced technology to Square D worldwide.

While many fortune 500 Companies are debating whether such programs should be centralized or decentralized, Square D CTC has conceived an implementation approach that may work for many other companies as well.

Within a short 8 months of existence, CTC has far exceeded its original goals in institutionalizing simulation methodologies in over 12 manufacturing locations. The acceptance and results have been overwhelming. The challenge ahead is to keep the momentum going, quantify benefits from such advanced practices, and, most of all, make simulation a 'way of life' on the plant floor.
\end{abstract}

\section{INTRODUCTION}

Our manufacturing environment today is complex and dynamic. To deal with this complexity, corporate manufacturing strategies are being established based on concepts such as JIT, synchronous manufacturing, time based manufacturing, and total quality management. In this environment, process simulation is being relied on more and more to help manufacturing management understand the implications of these concepts and for identifying and testing specific implementation strategies. The objective of this paper is to describe Square D Company's corporate simulation program and to outline the specific steps which were taken during its implementation.

This paper begins by describing the manufacturing environment at Square D Company and the motivation for using simulation. With this as a base, an eight-step approach for initiating and managing a corporate simulation program is described, and a summary of program results is included.

\section{BACKGROUND}

Square D is a worldwide supplier of products, systems, and services for the distribution, application, and control of electrical energy. The company currently operates approximately 55 manufacturing plants worldwide producing a variety of electrical distribution, control and sensing products. Over the last two to three years, Square D has used simulation to address issues relating to the specification and design of plant automation. But, each application was isolated and narrowly focused. In 1989, the Corporate Technology Center (CTC) was formed, with its charter to serve Square D by, among other things, institutionalizing modern/advanced design and manufacturing practices. Process and business simulation, synchronous manufacturing, time-based manufacturing, and activity based costing were identified as key practices which the CTC was to implement. The rest of this paper describes the specific implementation of the corporate simulation program.
The basis for Square D's simulation program comes from the recognition that the benefits of simulation fall into two categories: 1) those resulting from using a specific model and 2) those resulting from the practice of simulation methodology. Types of benefits falling into the first category are:

- Identification of cost avoidance during system specification and design

- Evaluation of operational strategies during system design

- Early evaluation of projected schedule/model mix

- Quantification of impacts of proposed system modifications and changes in manufacturing environment

(These types of benefits have traditionally been used to sell simulation.)

The second category of benefits are generally difficult to quantify but, in fact, can have a much greater impact than those in category one. This second type of benefit results from using simulation to increase plant involvement and to facilitate planning of the many aspects of designing and operating a manufacturing plant. Simulation provides a methodology for describing a plant system and forces a system-wide understanding. In order to "successfully" build any simulation model, many steps must be followed:

1. An objective must be defined.

2. The system must be described and flowcharted.

3. An appropriate level of detail must be selected and continually re-evaluated which will allow the user to meet the model objective.

4. Data must be gathered and analyzed which accurately reflects the system to be modeled.

5. The model must be built, verified and validated.

6. Experiments must be designed and conducted.

7. Documentation must be completed.

From Square D's perspective, the key aspect of this methodology is that it involves everyone-from plant manager to operator on the floor. In the Product/Process Design Phase, simulation supports the integration of manufacturing and engineering and thus supports simultaneous/concurrent engineering. In the System Design Phase and Operational Phase, simulation facilitates a team approach and creates a focus on system-wide optimization.

The objective of the simulation program was simply to maximize both types of benefits described above. To achieve this objective, three goals were set:

1. To have a self-sustaining simulation capability at the plant/divisional level.

2. To maximize involvement within each plant.

3. To have simulation become a standard practice ('way of life') within Square D.

With the objective and goals as a basis, an implementation plan was established. Throughout the past year, this plan has been revised and improved and will be described in the remainder of this paper. 


\section{PROGRAM IMPLEMENTATION}

\subsection{Implementation Approach}

The major steps which have been taken are listed below. Although the steps are listed in the order in which they were initially implemented as shown in Figure 1, all actions associated with each step are turning out to be on-going.

1. Obtain top management support

2. Select simulation software

3. Define roles/responsibilities

4. Obtain support at all levels in organization

5. Identify plant simulationists

6. Train plant simulationists

7. Train management

8. Implement projects with tangible results

(The most significant thing we are learning throughout the implementation of the simulation program is that nothing is a 'one shot deal', and that the program needs continual involvement from some central resource.)

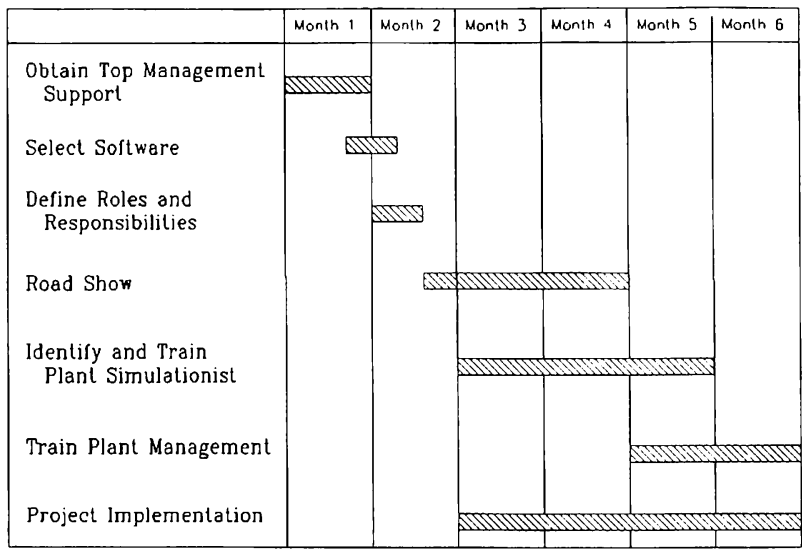

Figure 1. Corporate Simulation Program

\subsection{Obtain Top Management Support}

The initial and most critical step taken was soliciting our top management's support of the objective and goals for the proposed simulation program. The key factor which led to getting this up-front support was a lot of early involvement with top-level corporate management focused on developing the motivation for establishing a corporate-wide simulation program. The key point here is that contact with top management is not sufficient; they must be involved. In our case, the creation of the Corporate Technology Center played an important role in helping to get the necessary top-level involvement.

Over the past year we have seen how important this first step has been. Our experience leads us to believe that if top management is not involved up-front, the program will fizzle (i.e., 'simulation will be just another short-lived fad').

\subsection{Software Selection}

The intent of the simulation program is to establish simulation analysts in each plant. This presents a serious problem. Simulation tends to be as much art as science which generally means the need for dedicating a significant amount of time to the task to be effective. The plant environment however, tends to imply doing everything on an 'hour here, hour there' basis. So, it was critical that the selected software be structured in such a manner (i.e., menu driven) so as to minimize the 'art' aspect of modeling. As an added benefit, this would allow for more consistent modeling and would facilitate easy sharing of modeling techniques throughout the corporation. Also, the objective was to choose a package that allows the individual to quickly build basic models and yet have enough flexibility to allow for more complex (and realistic) models. Other key requirements were:

- manufacturing-specific terminology

- animation

- interactive model development and model experimentation

- PC based

- capability for models to be data driven

- vendor reputation for technical support

Given these objectives and software criteria, general simulation languages (i.e., SLAM, SIMAN, GPSS, etc.) were ruled out and several simulation packages (simulators) were evaluated. Based on the above criteria and requirements, WITNESS (AT\&T Istel) was selected.

\subsection{Roles and Responsibilities}

The three primary players in the simulation program are plant management, the plant simulationist, and the corporate simulation resource. It is important that each of these players has specific, well-defined responsibilities and is accountable for various aspects of the simulation program.

Management's role is to set the expectation for using simulation as a standard practice of system design and operation. More importantly, it is management that needs to be accountable for increasing the involvement in simulation at the local level. (We made an early mistake of expecting the plant simulationist to have this responsibility without them having the necessary authority over those needing to be involved.)

The plant simulationist's primary responsibility is project implementation and training of others. This individual has primary responsibility for increasing the plant understanding of simulation and for working with all groups in the plant to identify new applications.

The corporate simulation resource has responsibility to support management and the plant simulationists by ensuring that each has the appropriate training and on-going support to allow them to fulfill their responsibilities. The corporate resource must look beyond individual project implementations and focus on ensuring that each location is progressing towards becoming self-sustaining in simulation capability. The corporate resource is also responsible for leveraging learning and managing the network of simulationists throughout the corporation and for expanding application areas for simulation.

\subsection{Introduction of Simulation - Obtaining Support at ALL Levels}

As was described in the first step, the most critical support is that of top management. It is important to start at the top so that their support can be used to motivate involvement throughout the corporation.

In order to introduce the simulation program and generate support at the division and plant levels, a half-day 'road show' was developed. This presentation focused on the program goals and objectives, the 'ABC's of simulation (What, Why, When, Who), the roles and responsibilities defined above, and a handson demonstration of WITNESS. During this last part of the road show, an Istel representative led the group through a simple model building session focusing on a specific plant issue. This part of the road show proved to be an excellent method for initiating plant involvement in the simulation process and for highlighting the benefits which can be achieved as a result of simulation. It was also a good opportunity for building a relationship with the software vendor. 


\subsection{Plant Simulationist Identification}

The key to achieving the first goal of the simulation program (self-sustaining simulation capability at the plant/divisional level) is the selection of the individuals to be responsible for the plant simulation program. It is strongly recommended that more than one individual (one primary and at least one backup) be selected for each location. The existence of in-plant support is extremely beneficial and, many times necessary, when modeling most systems. Also, identifying and training a backup prevents loosing the plant's entire simulation capability due to a future change in personnel. The process of selection should focus on several personal attributes.

- Some degree of computer literacy. (Enough to be comfortable with hardware, operating system, and software applications.)

- Good manufacturing background with knowledge of the key plant personnel and an understanding of the plant decision making process.

- Strong interpersonal skills are essential to be able to interface credibly with all groups in the plant.

- Programming experience is a plus.

Once the individuals are selected, it is important that a formal role be developed and that both the individuals and their immediate management make this role a significant part (50 to 80 percent) of their performance criteria. This is important for establishing accountability for the success of the simulation program at the local level. The corporate resource should provide the skeleton role and assist with making it fit the specific plant situation. This will help to guarantee consistency of responsibility and expectation across the corporation.

\subsection{Simulationist Training}

The training program covers general simulation issues (What is simulation? Why use simulation? When should simulation be used?), simulation methodology, formal WITNESS training, statistical issues and techniques, and hands-on project work. Training resources included several books on simulation and related subjects, Istel's WITNESS class, and the corporate resource.

As a general rule, the first two to three months need to be allocated for training with at least the primary simulationist dedicating 80 to 100 percent of the time to the training. It is important that the objective during this period is training only, and that any commitments of providing model results be avoided.

The formal training period lasts four to six weeks and consists of four phases which are shown in Figure 2.

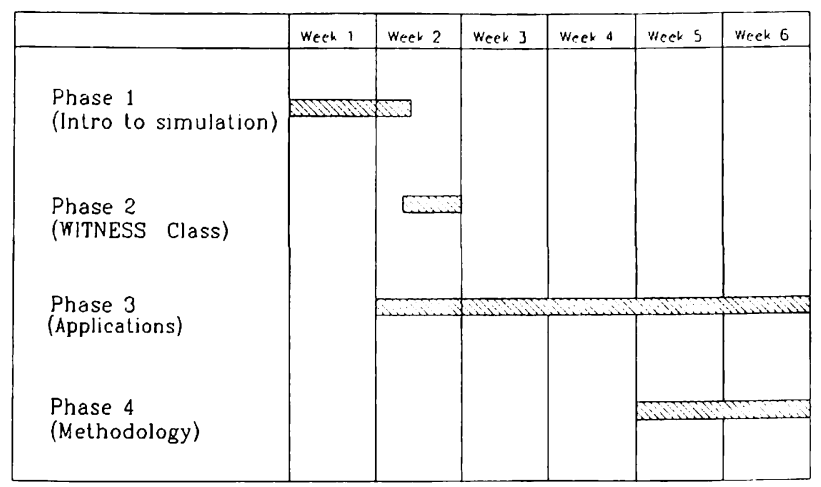

Figure 2. Plant Simulationist Training
Phase 1 consists of time spent with the corporate resource focusing on general simulation issues and a basic hands-on introduction to WITNESS which helps the individuals maximize their phase 2 training. During phase 1, simulation project methodology is described and the roles and responsibilities are discussed. Also, several small plant applications are identified and model scope and objectives are defined. class.

Phase 2 consists of Istel's three to four day WITNESS

Phase 3 consists of attempting to model the applications identified during phase 1 . Generally this phase lasts two to three weeks. The corporate resource may be involved directly during this phase but indirect (phone) support is preferred. This allows the plant simulationist to get their hands 'dirty' and learn WITNESS.

Phase 4 involves direct contact with the corporate resource and generally lasts a week. The focus of this phase is methodology, advanced WITNESS training relative to the applications worked on in phase 3, and basic statistics as they relate to simulation (i.e., data analysis, distribution characteristics, the effects of random numbers, determination of warm-up period and run length/number of replications, recording and interpretation of model outputs).

On-going support from the corporate resource has been essential to ensure that the development of the plant simulationists continues beyond the formal training period. After this initial training, the focus shifts to application-specific needs along with more in-depth training on statistical techniques and design of experiments. At this point, the corporate resource becomes more of an internal consultant involved in project identification, model initiation, project trouble-shooting, and support of model experimentation and analysis.

\section{MANAGEMENT TRAINING}

Referring back to the roles and responsibilities described earlier, it is unrealistic in most cases to expect management to handle their responsibilities without the appropriate support and training. First and foremost, management must be convinced that the benefits of simulation exist. Secondly, they must understand the methodology behind simulation and the importance of getting all the appropriate people involved.

Management training has taken several different formats ranging from the initial road show described earlier to a formal one day hands-on workshop. Regardless of the format taken, as much one-on-one contact as possible is very important to address any of the specific concerns and questions that arise.

The most successful format for management training has been a one day workshop involving the plant manager and staff. The workshop focuses on the issues directly related to maximizing the benefits of simulation. The major part of the workshop is using a model or model segment from their plant to perform 'what if' analysis. This hands-on format allows the management teams to get a working knowledge of using simulation to address problems they deal with on a daily basis. An example best illustrates this format.

An assembly group was modeled as it existed in the manufacturing plant. Using the model, the group was taught how to make specific modifications to the model (i.e., adding capacity, removing buffer, changing staffing levels, changing setup, cycle, and downtime). Real costs were associated with the various modifications and dollar savings were associated with reductions in inventory and lead-time, and increases in throughput. The group was then allowed to perform actual hands-on analysis to improve the model performance and quantify the results in terms of dollars. As a result of the day, the management team got a real appreciation of the benefits (both tangible - dollars, and intangible - teamwork, improved problem solving) which could be achieved through simulation. 


\section{PROGRAM RESULTS}

How well has this approach worked? Eight months after initiation of the simulation program, the following corporate network has been established. Full-time simulation roles have been created at nine plant locations with four to six other locations considering creating a role. Approximately 20 people have been trained in the simulation methodology and in WITNESS. Specific benefits are being realized in many of our plants:

- Cost avoidance is being realized on new systems design as a result of simulation analysis.

- Simulation is being used successfully to support the integration of engineering and manufacturing on most new product programs. On several of Square D's current major programs, the project team includes a dedicated simulationist.

- Several plants, after only 6 months, are noticing significant increases in plant involvement in operational analysis and support of plant JIT implementation.

- Simulation is being used to address non-manufacturing applications such as the consolidation of the corporate customer service center.

- Divisional standards have been set requiring simulation analysis as part of all major systems design and prior to major capital expenditures.

\section{SUMMARY}

Process simulation is proving to be a valuable tool and methodology for allowing our plants to define and evaluate their manufacturing strategies and for increasing plant involvement in the planning process. Simulation is helping the plants quantify the impacts of implementing these strategies.

As a company, Square D is beginning to meet the goals of the simulation program.

- We are establishing a self-sustaining simulation capability at the plant level.

- Plant involvement in the modeling process is increasing.

- Process simulation is becoming a standard practice in our divisions and their associated manufacturing plants.

These program successes, achieved in less than a year, reflect the effectiveness of the eight-step implementation approach (shown in Figure 1) which has been described in this paper. In summary, these steps are:

1. Obtain top management support

2. Select simulation software

3. Define roles/responsibilities

4. Obtain support at all levels in organization

5. Identify plant simulationists

6. Train plant simulationists

7. Train management

8. Implement projects with tangible results

We strongly recommend close consideration of this approach for making simulation a standard practice in other companies. 